\title{
Bhabha scattering at LEP1. Calorimeter event selection
}

\author{
G.I.Gakh, V.Yu.Gonchar, N.P.Merenkov \\ National Scientific Centre "Kharkov Institute of Physics and Technology" \\ Akademicheskaya 1, 310108, Kharkov, Ukraine
}

\begin{abstract}
Analytical calculations have been used to describe the calorimeter event selection in small-angle electronpositron scattering at LEP1. The first-order QED correction to the Born cross-section has been derived with leading log and next-to-leading log approximations. The second-- and third-order corrections are computed with leading accuracy. Our analytical results are illustrated by Tables and compared with the corresponding results obtained with the of Monte Carlo generator BHLUMI.
\end{abstract}

PACS 12.15.Lk, 12.20.-m, 12.20.Ds, 13.40.-f 


\section{Introduction}

The test of the Standard Model (SM) based on the unified theory of electroweak interaction and quantum chromodynamics, is one of the primary aims of experiments at LEP1 and LEP2. The small-angle Bhabha scattering (SABH) process is used to measure the luminosity of the corresponding electron-positron collider. Being the normalization factor, the cross-section for the SABH process affects all observable cross-sections and is a significant component in both the precision measurements of the SM parameters and investigation of new physics at LEP1 [1].

By now a purely experimental precision better than 0.1 percent for the luminosity determination has been achieved at LEP1 [2]. However, to obtain the total value a systematic theoretical error must be added. Ths latter is determined by the accuracy of theoretical description of SABH cross-section, that takes into consideration the specidic features of event selection and the detector geometry at LEP1. Therefore, there is a need to decrease its precision to the level of experimental one or better it. It is no mere chance that lately much attention has been given to the theoretical investigation of the SABH process [3-14].

The theoretical calculation of the SABH cross-section at LEP1 involves two somewhat different tasks. The first one is to privide an adequate description of the experimental restrictions imposed on the event selection in terms of final-particle phase space. The second task consists in writing the matrix element squared within the required accuracy. There are two methods for theoretical investigation of SABH at LEP1: the method based on the Monte Carlo event generator [3-6] and the method resting on semianalytical calculations [7-14].

The advantage of the Monte Carlo method is that it enables simulation of detectors and event selection schemes of different types. The modern Monte Carlo generators employ some additional procedures (Yennie-Frautchi-Suura exponentiation [15], electron structure function method [16] and effective-coupling-constant approach [17]) to eliminate problems associated with the infrared divergence, and to take into account the leading corrections in the higher orders of the perturbation theory. Although a part of the second-order next-to-leading correction 
is compensated due to the exponential form of the electron structure function (see, for example, [7]), the total second-order correction, including $\alpha^{2} \ln \left(Q^{2} / m^{2}\right)$ terms (here $Q^{2}$ is the typical value of the transfer momentum squared), remains undeterminated.

The advantage of the analytical method is that it allows the use of the exact matrix element squared, based on essential Feynman diagrams. The infrared problem in the context of this approach can be solved in the usual way by taking into account virtual, real (soft and hard) photon emission and pair production in every order of the perturbation theory. The shortcoming of the analytical approach is its low mobility with respect to the change in the experimental conditions used for the event selection. Nevertheless, the analytical calculations are very important because they privide checking many Monte Carlo calculations for different "ideal" detectors.

At present, four types of "ideal" event selections are used for the comparison of various $\mathrm{MC}$ - generators. One of them is completely inclusive as to the final electron and positron. In this case, the event is the simultaneous detection of the scattered electron and positron with the invariant mass higher than a certain threshold value. The ring-shaped symmetric or asymmetric detectors are used for the detection. this method of event selection is called in Ref.[3] as BARE1.

The other three methods of event selection, called in Ref.[3] as CALO1, CALO2 and SICAL2, are calorimetric. If the final particles (photons, electrons and positrons) move almost in parallel to each other, i.e., they form a cluster, then the total energy of all particles in the cluster is determined at the calorimeter event selection. If the final particles do not form a cluster, then CALO1 and CALO2 detect only the scattered electron and positron, whereas SICAL2 does not discriminate photons and electrons (or positrons). CALO1 and CALO2 differ in the shape and size of the cluster. The detectors of the same geometry as in the case of BARE1 are used particle detection.

It is most natural to apply the analytical approach to the same methods of the event selection, which are used in the $\mathrm{MC}$-generators. The analytical formulae for the SABH cross-section at LEP1 were published for the BARE1 method of event 
selection in the cases of symmetric [11-13] and asymmetric [14] detectors. Those formulae include the full first-order correction as well as the second-order correction with leading and next-to-leading accuracy. They also include the thirdorder correction in the leading approximation. It is just these contributions that must be calculated in order to achieve the required accuracy. Some brief analytical results for the CALO1 and CALO2 event selections in the case of symmetric detectors have been given in Ref.[14,25].

In this paper we present full analytical calculations of the SABH cross-section at LEP1 for CALO1 and CALO2 in the most general case of asymmetric detectors. The corresponding formulae include the same contributions as for the BARE1 method, except the second-order next-to-leading contribution. As to the SICAL2 method, we hope to investigate it in the following publications.

The paper is organized as follows. In Section 2 we introduce the "observable" cross-section $\sigma_{o b s}$ with allowance for the experimental restrictions on the angles and energies of the detected particles. We also calculate the first-order correction for the BARE1 method in the case of asymmetric detectors. The formulae obtained are widely used below. In Section 3 the first-order correction is considered for the CALO1 method, and in Section 4 - for the CALO2. In Section 5 we present the formulae for the leading contributions in the second and third orders using the electron structure function method. These formulae are universal because they are independent of the shape and size of the cluster. In Section 6 we present the Tables that illustrate the comparison of our analytical results with the corresponding calculations made with the MC-generator BHLUMI [3].

\section{First-order correction for BARE1}

For the investigation of radiative corrections to the Born cross-section of SABH at LEP1 it is convenient to introduce the dimensionless quantity

$$
\Sigma=\frac{1}{4 \pi \alpha^{2}} Q_{1}^{2} \sigma_{o b s}
$$

where $Q_{1}^{2}=\varepsilon^{2} \theta_{1}^{2}$ ( $\varepsilon$ is the beam energy, and $\theta_{1}$ is the minimal angle of the wide detector). The "observed" cross-section $\sigma_{o b s}$ is determined by the following 
formula in the BARE1 case

$$
\sigma_{o b s}=\int d x_{1} d x_{2} \Theta d^{2} q_{1}^{\perp} d^{2} q_{2}^{\perp} \Theta_{1}^{c} \Theta_{2}^{c} \frac{d \sigma\left(e^{+}+e^{-} \rightarrow e^{+}+e^{-}+X\right)}{d x_{1} d x_{2} d^{2} q_{1}^{\perp} d^{2} q_{2}^{\perp}},
$$

where $\mathrm{X}$ denotes undetected final particles, and $x_{1}\left(x_{2}\right)$ and $\vec{q}_{1}^{\perp}\left(\vec{q}_{2}^{\perp}\right)$ are the energy fraction and the transverse component of the momentum of the electron (positron) in the final state. The functions $\Theta_{i}^{c}(i=1,2)$ take into account the angular cuts, and the function $\Theta$ takes into account the cutoff on the energies of the detected electron and positron

$$
\begin{gathered}
\Theta_{1}^{c}=\theta\left(\theta_{3}-\theta_{-}\right) \theta\left(\theta_{-}-\theta_{1}\right), \quad \Theta_{2}^{c}=\theta\left(\theta_{4}-\theta_{+}\right) \theta\left(\theta_{+}-\theta_{2}\right), \quad \Theta=\theta\left(x_{1} x_{2}-x_{c}\right), \\
\theta_{-}=\frac{\left|\vec{q}_{1}^{\perp}\right|}{x_{1} \varepsilon}, \quad \theta_{+}=\frac{\left|\vec{q}_{2}^{\perp}\right|}{x_{2} \varepsilon} .
\end{gathered}
$$

In the case of asymmetrical ring-shaped detectors we have

$$
\theta_{3}>\theta_{4}>\theta_{2}>\theta_{1}, \quad \rho_{i}=\frac{\theta_{i}}{\theta_{1}}>1 .
$$

Below we shall assume, for definiteness, that the final electron is registered by wide detector $\left(\theta_{3}, \theta_{1}\right)$, and positron - by narrow one $\left(\theta_{4}, \theta_{2}\right)$.

The Born contribution to $\Sigma$, for SABH process at LEP1, is determined as follows

$$
\Sigma_{B}=\int_{\rho_{2}^{2}}^{\rho_{4}^{2}} \frac{d z}{z^{2}}\left(1-\frac{z}{2} \theta_{1}^{2}\right),
$$

and in symmetrical case the limits of integration are $\left(\rho_{3}^{2}, 1\right)$. The Born crosssection is the same both for the inclusive and calorimeter event selections. Formula (4) includes the contribution of the scattering diagram and its interference with the annihilation one. The contribution of the annihilation diagram is proportional to $\theta_{1}^{4}$ and may be omitted within the scope of $0.1 \%$. When calculating the radiative corrections to the cross-section (4), we systematically omit the terms proportional to $\theta_{1}^{2}$.

The first-order correction to the cross-section of electron-positron scattering includes the contributions connected with radiation of the virtual and real (soft and hard) photons and vacuum polarization due to the light fermions including hadrons. The contribution of vacuum polarization is universal, and therefore we shall not take it into account below.

$$
\Sigma_{1}=\Sigma_{V+S}+\Sigma_{H}+\Sigma^{H} .
$$


The correction due to the virtual and soft real photon emission (with the energy less than $\Delta \varepsilon, \Delta \ll 1$ ) can be written as follows [12] (in this case $x_{1}=x_{2}=$ $\left.1, \quad \vec{q}_{1}^{\perp}+\vec{q}_{2}^{\perp}=0\right)$

$$
\Sigma_{V+S}=2 \frac{\alpha}{\pi} \int_{\rho_{2}^{2}}^{\rho_{4}^{2}} \frac{d z}{z^{2}}\left[2(L-1) \ln \Delta+\frac{3}{2} L-2\right], \quad L=\ln \frac{\epsilon^{2} \theta_{1}^{2} z}{m^{2}},
$$

where $z=\vec{q}_{2}^{\perp} / Q_{1}^{2}, m$ is the electron mass.

The second term on the right side of Eq.(5) is due to the emission of the hard( with energy greater than $\Delta \varepsilon$ ) photon by the positron registered by the narrow detector. In this case we have

$$
X=\gamma\left(\vec{k}^{\perp}, 1-x_{2}\right), \quad x_{1}=1, \vec{k}^{\perp}+\vec{q}_{1}^{\perp}+\vec{q}_{2}^{\perp}=0, \quad x_{c}<x_{2}<1-\Delta,
$$

where $\vec{k}^{\perp}$ is the transverse component of the three-momentum of the hard photon. The corresponding contribution can be obtained by the integration of the bremsstrahlung differential cross-section in the electron-positron collision over the region

$$
1<z<\rho_{3}^{2}, \quad x^{2} \rho_{2}^{2}<z_{1}=\frac{\vec{q}_{1}^{\perp 2}}{Q_{1}^{2}}<x^{2} \rho_{4}^{2}, \quad-1<\cos \varphi<1,
$$

where $\varphi$ is the angle between two-dimensional vectors $\vec{q}_{1}^{\perp}$ and $\vec{q}_{2}^{\perp}$. The result is defined by the formula

$$
\Sigma_{H}=\frac{\alpha}{2 \pi} \int_{1}^{\rho_{3}^{2}} \frac{d z}{z^{2}} \int_{x_{c}}^{1-\Delta} \frac{1+x^{2}}{1-x} d x\left[(L-1)\left(\Delta_{42}+\Delta_{42}^{(x)}\right)+K\left(x, z ; \rho_{4}, \rho_{2}\right)\right],
$$

where

$$
\begin{gathered}
\widetilde{K}=\frac{(1-x)^{2}}{1+x^{2}}\left(\Delta_{42}+\Delta_{42}^{(x)}\right)+\Delta_{42} \widetilde{L}_{1}+\Delta_{42}^{(x)} \widetilde{L}_{2}+\left(\bar{\theta}_{4}^{(x)}-\theta_{2}^{(x)}\right) \widetilde{L}_{3}+\left(\bar{\theta}_{4}-\theta_{2}\right) \widetilde{L}_{4}, \\
\widetilde{L}_{1}=\ln \left|\frac{\left(z-\rho_{2}^{2}\right)\left(\rho_{4}^{2}-z\right) x^{2}}{\left(x \rho_{4}^{2}-z\right)\left(x \rho_{2}^{2}-z\right)}\right|, \quad \widetilde{L}_{2}=\ln \left|\frac{\left(z-x^{2} \rho_{2}^{2}\right)\left(x^{2} \rho_{4}^{2}-z\right)}{x^{2}\left(x \rho_{4}^{2}-z\right)\left(x \rho_{2}^{2}-z\right)}\right| \\
\widetilde{L}_{3}=\ln \left|\frac{\left(z-x^{2} \rho_{2}^{2}\right)\left(x \rho_{4}^{2}-z\right)}{\left(x^{2} \rho_{4}^{2}-z\right)\left(x \rho_{2}^{2}-z\right)}\right|, \quad \widetilde{L}_{4}=\ln \left|\frac{\left(z-\rho_{2}^{2}\right)\left(x \rho_{4}^{2}-z\right)}{\left(\rho_{4}^{2}-z\right)\left(x \rho_{2}^{2}-z\right)}\right|
\end{gathered}
$$

and the following designations are used for $\theta$ - functions:

$$
\begin{gathered}
\Delta_{42}^{(x)}=\theta_{4}^{(x)}-\theta_{2}^{(x)}, \quad \Delta_{42}=\theta_{4}-\theta_{2} \\
\theta_{i}^{(x)}=\theta\left(x^{2} \rho_{i}^{2}-z\right), \quad \theta_{i}=\theta\left(\rho_{i}^{2}-z\right), \quad \bar{\theta} i^{(x)}=1-\theta_{i}^{(x)}, \quad \bar{\theta}_{i}=1-\theta_{i} .
\end{gathered}
$$


The term $\Sigma^{H}$, related to the emission of the hard photon by electron registered by means of the wide detector, can be obtained from (9) by change of the integration limits over $z:\left(\rho_{4}^{2}, \rho_{2}^{2}\right)$ instead of $\left(\rho_{3}^{2}, 1\right)$ and substitution

$$
\Delta_{42} \rightarrow 1, \quad \Delta_{42}^{(x)} \rightarrow \theta_{3}^{(x)}, \quad K\left(x, z ; \rho_{4}, \rho_{2}\right) \rightarrow K\left(x, z ; \rho_{3}, 1\right)
$$

in the integrand. It is easy to see that the contribution, including the analogue of $\widetilde{L}_{4}$, vanishes, and it is caused by the specific character of the accompanying $\theta$ - functions.

The individual parts on the right side of Eq.(5) depend on the auxiliary parameter $\Delta$, but this dependence disappeares in the sum, so that the first-order correction can be written as follows

$$
\begin{aligned}
& \Sigma_{1}=\frac{\alpha}{2 \pi}\left\{\int_{1}^{\rho_{3}^{2}} \frac{d z}{z^{2}} \int_{x_{c}}^{1}\left[\left(-\frac{1}{2} \delta(1-x)+(L-1) P_{1}(x)\right)\left(\Delta_{42}+\Delta_{42}^{(x)}\right)+\frac{1+x^{2}}{1-x} K\left(x, z ; \rho_{4}, \rho_{2}\right)\right] d x\right. \\
& \left.+\int_{\rho_{2}^{2}}^{\rho_{4}^{2}} \frac{d z}{z^{2}} \int_{x_{c}}^{1}\left[\left(-\frac{1}{2} \delta(1-x)+(L-1) P_{1}(x)\right)\left(1+\theta_{3}^{(x)}\right)+\frac{1+x^{2}}{1-x} K\left(x, z ; \rho_{3}, 1\right)\right] d x\right\}, \quad(13)
\end{aligned}
$$

where $P_{1}(x)$ determines the iterative form of the nonsinglet electron structure function (see, for examle, Ref.[7])

$$
P_{1}(x)=\frac{1+x^{2}}{1-x} \theta(1-x-\Delta)+\left(2 \ln \Delta+\frac{3}{2}\right) \delta(1-x), \quad \Delta \rightarrow 0 .
$$

In order to make the cancellation of the $\Delta$ - dependence on the right side of Eq.(10) more transparent, one may use the following relations

$$
\begin{gathered}
\int_{x_{c}}^{1} P_{1}(x) d x=-\int_{0}^{x_{c}} \frac{1+x^{2}}{1-x} d x, \quad \int_{x_{c}}^{1} P_{1}(x) \bar{\theta}_{3}^{(x)} d x=\bar{\theta}_{3}^{\left(x_{c}\right)} \int_{x_{c}}^{\sqrt{z} / \rho_{3}} \frac{1+x^{2}}{1-x} d x, \\
\int_{x_{c}}^{1} P_{1}(x) \bar{\Delta}_{42}^{(x)} d x=\theta_{4} \bar{\theta}_{4}^{\left(x_{c}\right)} \int_{x_{c}}^{\sqrt{z} / \rho_{4}} \frac{1+x^{2}}{1-x} d x-\theta_{2} \bar{\theta}_{2}^{\left(x_{c}\right)} \int_{x_{c}}^{\sqrt{z} / \rho_{2}} \frac{1+x^{2}}{1-x} d x, \\
\bar{\Delta}_{42}^{(x)}=\Delta_{42}-\Delta_{42}^{(x)} .
\end{gathered}
$$

As we already noted, the right side of formula (13) defines the first-order correction to the Born cross-section of SABH at LEP1 with switched off vacuum polarization. The last one can be included by inserting the quantity $\left[1-\Pi\left(z Q_{1}^{2}\right)\right]^{-2}$ in the integrand (as to $\Pi$ see Ref. [3] and the bibliography cited there). 
The upper line on the right side of Eq.(13) corresponds to the emission of the real and virtual photons by a positron and lower one - by an electron. Besides, the terms that are accompanied by the $x$-depending ( $x$-independing) $\theta$ - functions describe the emission in the initial (final) state. This information is very important for the investigation of calorimeter event selection.

But before to move on this investigation, we note one more circumstance. When deriving the Eq.(13), we systematically ignore the terms of the order of $\theta^{2} \approx Q^{2} / S(S=4 \varepsilon)$ in comparison with a unity. However, as it is known [18], the terms of this type have a double-logarithmic asymptotics. Their contribution to $\Sigma_{1}$ equals parametrically to $[12,19]$

$$
\frac{\alpha}{\pi} \frac{Q^{2}}{S} \ln ^{2} \frac{Q^{2}}{S},
$$

and it amounts to about $10^{-4}$ in the LEP1 conditions. Therefore, we expect the systematic deviation of our calculations in the first order of the perturbation theory from the corresponding results of MC-generator BHLUMI, which completely includes such contributions on the $0.01 \%$ level (see Section 6).

\section{Cone-shaped cluster CALO1}

In the case of calorimeter event selection the detector does not discriminate the particles moving near the same direction, i.e. producing a cluster. In the first order of perturbation theory the cluster can be formed only by two particles: photon and electron (or photon and positron). For the definiteness we shall talk about the positron cluster, although the same will be refer to the electron one.

If a photon and positron belong to a cluster $\left(\gamma, \mathbf{e}^{+}\right) \in \mathbf{C L}$, the detector measures the total energy of the cluster, and its position is determined by the positron position. If a photon and positron do not form a cluster $\left(\gamma, \mathbf{e}^{+}\right) \notin \mathbf{C L}$, the event looks exactly the same as in the case of BARE1, i.e. there exists the detection threshold on the positron energy and the photon is entirely not detected. The cutoff on the positron energy can be written symbolically in the form

$$
\int_{x_{c}}^{1} d x+\int_{0}^{x_{c}}\left[\left(\gamma, e^{+}\right) \in C L\right] d x \equiv \int_{0}^{1} d x-\int_{0}^{x_{c}}\left[\left(\gamma, e^{+}\right) \notin C L\right] d x .
$$


It is convenient to use the left side of Eq.(14) in order to take into account the initial-state emission and right side of Eq.(14) for the description of the final-state one. As follows from Eq.(14), in the calorimeter event selection the correction to the cross-section can be represented in the form of two-term sum. One of these terms is a universal, i.e., it does not depend on the cluster specific and another one is determined by its form and size

$$
\Sigma_{1}=\Sigma_{1}^{u n}+\Sigma_{1}^{c l}
$$

The contribution of the universal part $\Sigma_{1}^{u n}$ can be obtained with the help of the formula (13). For this one must retain without change the correction caused by the initial-state emission and integrate the correction, caused by the final-state emission, for $x$ going from 0 to 1 . The result has the following form

$$
\begin{gathered}
\Sigma_{1}^{u n}=\frac{\alpha}{2 \pi} \int_{1}^{\rho_{3}^{2}} \frac{d z}{z^{2}}\left\{-\Delta_{42}^{(x)}+\int_{x_{c}}^{1}\left[\left((L-1) P_{1}(x)+1-x+\frac{1+x^{2}}{1-x} \widetilde{L}_{2}\right) \Delta_{42}^{(x)}+\left(\bar{\theta}_{4}^{) x)}-\theta_{2}^{(x)}\right) \widetilde{L}_{3}\right] d x+\right. \\
\left.+\int_{0}^{1}\left[\left(1-x+\frac{1+x^{2}}{1-x} \widetilde{L}_{1}\right) \Delta_{42}+\frac{1+x^{2}}{1-x}\left(\bar{\theta}_{4}-\theta_{2}\right) \widetilde{L}_{4}\right] d x\right\}+ \\
+\frac{\alpha}{2 \pi} \int_{\rho_{2}^{2}}^{\rho_{4}^{2}} \frac{d z}{z^{2}}\left\{-1+\int_{x_{c}}^{1}\left[\left((L-1) P_{1}(x)+1-x+\frac{1+x^{2}}{1-x} L_{2}\right) \theta_{3}^{(x)}+\bar{\theta}_{3}^{(x)} L_{3}\right] d x+\right. \\
\left.+\int_{0}^{1}\left(1-x+\frac{1+x^{2}}{1-x} L_{1}\right) d x\right\}
\end{gathered}
$$

where $L_{i}$ are obtained from $\widetilde{L}_{i}$ by the substitution $\rho_{3}$ instead of $\rho_{4}$ and 1 instead of $\rho_{2}$. In the case of symmetrical ring-shaped detectors it is necessary to put $\rho_{3}=\rho_{4}, \rho_{2}=1$ on the right side of Eq.(16).

We see that owing to the well-known relation

$$
\int_{0}^{1} P_{1}(x)=0,
$$

the final-state emission does not give rise to large $\operatorname{logarithm} L$ in the cross-section in accordance with the Lee-Nauenberg theorem about the cancellation of the mass singularities when summing over all possible states [21].

In order to obtain the part of the correction, determined by the shape and size of the cluster, one may use the simplified form of the bremsstrahlung differential cross-section, that describes the so-called semicollinear kinematics (see, for 
example, Refs. $[14,22])$. In the case of single-photon emission it simply means, that one can neglect the electron mass everywhere. The corresponding expression for the dimensionless quantity $\Sigma$, related to the photon emission by positron, is determined as follows

$$
d \Sigma=\frac{\alpha d \varphi d z d z_{1}\left(1+x^{2}\right)}{4 \pi Q_{1}^{2} z\left(z_{1}-x z\right)}\left[\frac{1}{z_{1}+z+2 \sqrt{z_{1} z} \cos \varphi}-\frac{x}{z_{1}+x^{2} z+2 x \sqrt{z_{1} z} \cos \varphi}\right] d x .
$$

The cutoff on the $z, z_{1}$ and $\varphi$ variables depends, of course, on the cluster shape. The CALO1 cluster is the cone with the cone semi-angle $\delta=0.01$ around the final-positron 3-momentum. In order to include the initial-state emission it is necessary to have (according to Eq. (14)) the cutoff on these variables when photon and positron belong to a cluster

$\left|\sqrt{z_{1}}-x \sqrt{z}\right|<x(1-x) \lambda,-1<\cos \varphi<-1+\frac{\lambda^{2} x^{2}(1-x)^{2}-\left(\sqrt{z_{1}}-x \sqrt{z}\right)^{2}}{2 x \sqrt{z z_{1}}}, \quad \lambda=\frac{\delta}{\theta_{1}}$

For the calculation of the correction caused by the final-state emission it is necessary to use the cutoff when photon and positron do not form a cluster

$$
\begin{gathered}
-1<\cos \varphi<1, \quad\left|\sqrt{z_{1}}-x \sqrt{z}\right|>\lambda x(1-x) \\
1>\cos \varphi>-1+\frac{\lambda^{2} x^{2}(1-x)^{2}-\left(\sqrt{z_{1}}-x \sqrt{z}\right)^{2}}{2 x \sqrt{z z_{1}}}, \quad\left|\sqrt{z_{1}}-x \sqrt{z}\right|<\lambda x(1-x) .
\end{gathered}
$$

We pay attention to the fact, that formally the cuts (18) and (19) determine also the collinear and semicollinear kinematic regions, respectively (in the case of the final-state emission $[12,13,22])$. The only, but very essential difference is that, in the considered case, the parameter $\lambda$ is of the order of unity, whereas when determining the kinematic regions, it is required that this parameter was much less than unity.

Integration of Eq.(17), as well as a similar formula including photon emission by an electron, over regions (18) and (19) leads to the following contribution to the first-order correction

$$
\begin{gathered}
\Sigma_{1}^{c l}=\Sigma_{1 i}^{c l}+\Sigma_{1 f}^{c l} \\
\Sigma_{1 i}^{c l}=\frac{\alpha}{2 \pi} \int_{0}^{x_{c}} \frac{1+x^{2}}{1-x} d x \int \frac{d z}{z^{2}} \int d z_{1}(\Psi+\widetilde{\Psi}) \Phi\left(z_{1}, z ; \lambda, x\right),
\end{gathered}
$$




$$
\begin{aligned}
& \Sigma_{1 f}^{c l}=\frac{\alpha}{2 \pi} \int_{0}^{x_{c}} \frac{1+x^{2}}{1-x} d x\left\{\int_{a_{0}^{2}}^{b^{2}} \frac{d z}{z^{2}}\left(\ln \left|\frac{x \rho_{3}^{2}-z}{\rho_{3}^{2}-z}\right|+l_{+}\right)+\int_{a^{2}}^{b_{0}^{2}} \frac{d z}{z^{2}}\left(\ln \left|\frac{x-z}{1-z}\right|+l_{-}\right)+\right. \\
& +\int_{1}^{\rho_{3}^{2}} \frac{d z}{z^{2}}\left[\theta\left(\tilde{a}_{0}^{2}-z\right)-\theta\left(z-\tilde{b}_{0}^{2}\right)\right] \tilde{L}_{4}+\int_{\tilde{a}_{0}^{2}}^{\tilde{b}^{2}} \frac{d z}{z^{2}}\left(\ln \left|\frac{x \rho_{4}^{2}-z}{\rho_{4}^{2}-z}\right|+l_{+}\right)+\int_{\tilde{a}_{0}^{2}}^{\tilde{b}_{0}^{2}} \frac{d z}{z^{2}}\left(\ln \left|\frac{x \rho_{2}^{2}-z}{\rho_{2}^{2}-z}\right|+l_{-}\right)+ \\
& \left.\left.\quad+\int \frac{d z}{z^{2}} \int d z_{1}(\Psi+\widetilde{\Psi}) F\left(z_{1}, z ; \lambda, x\right)\right\}, \quad l_{ \pm}=\ln \frac{\lambda[2 \sqrt{z} \pm \lambda(1-x)]}{z \mp 2 x \lambda \sqrt{z}-\lambda^{2} x(1-x)}, \quad, \quad 21\right)
\end{aligned}
$$

where the integration limits over $z$ (in the squared brackets) and $z_{1}$ (in the round brackets) are defined with the help of $\Psi$ and $\widetilde{\Psi}$ quantities

$$
\begin{gathered}
\Psi=\left[a^{2}, a_{0}^{2}\right]\left(x^{2} z_{+}, x^{2}\right)+\left[b^{2}, a^{2}\right]\left(x^{2} z_{+}, x^{2} z_{-}\right)+\left[b_{0}^{2}, b^{2}\right]\left(x^{2} \rho_{3}^{2}, x^{2} z_{-}\right), \quad z_{ \pm}=(\sqrt{z} \pm \lambda(1-x))^{2}, \\
\widetilde{\Psi}=\left[\tilde{a}^{2}, \tilde{a}_{0}^{2}\right]\left(x^{2} z_{+}, x^{2} \rho_{2}^{2}\right)+\left[\tilde{b}^{2}, \tilde{a}^{2}\right]\left(x^{2} z_{+}, x^{2} z_{-}\right)+\left[\tilde{b}_{0}^{2}, \tilde{b}^{2}\right]\left(x^{2} \rho_{4}^{2}, x^{2} z_{-}\right), \\
z_{ \pm}=(\sqrt{z} \pm \lambda(1-x))^{2}, \quad a_{0}=\rho_{2}, \quad b_{0}=\rho_{4}, \quad a=\max \left(\rho_{2}, 1+\lambda(1-x)\right), \\
b=\min \left(\rho_{4}, \rho_{3}-\lambda(1-x)\right), \quad \tilde{a}_{0}=\max \left(1, \rho_{2}-\lambda(1-x)\right), \quad \tilde{a}=\rho_{2}+\lambda(1-x), \\
\tilde{b}=\rho_{4}-\lambda(1-x), \quad \tilde{b}_{0}=\min \left(\rho_{4}+\lambda(1-x), \rho_{3}\right) .
\end{gathered}
$$

The $\Phi$ and $F$ functions beloning to the right side of Eq.(21) are determined as follows:

$$
\begin{gathered}
\Phi=\frac{2}{\pi}\left(\frac{1}{z_{1}-x z}+\frac{1}{z-z_{1}}\right) \arctan \left\{\frac{z-z_{1}}{\left(\sqrt{z}-\sqrt{z_{1}}\right)^{2}} R\right\}, \\
F=\frac{2}{\pi}\left(\frac{1}{z_{1}-x z}-\frac{1}{z_{1}-x^{2} z}\right) \arctan \left\{\frac{\left(\sqrt{z_{1}}-x \sqrt{z}\right)^{2}}{z_{1}-x^{2} z} R^{-1}\right\}, \\
R=\sqrt{\frac{\lambda^{2} x^{2}(1-x)^{2}-\left(\sqrt{z_{1}}-x \sqrt{z}\right)^{2}}{\left(\sqrt{z_{1}}+x \sqrt{z}\right)^{2}-\lambda^{2} x^{2}(1-x)^{2}}} .
\end{gathered}
$$

The formula (21) is valid in the general case of the asymmetrical detectors . In the symmetrical case of the wide detectors it is necessary to put $\rho_{4}=\rho_{3}$ and $\rho_{2}=1$ in Eq. $(21)$

\section{Pyramidal cluster CALO2}

The cluster CALO2 has a pyramidal form. Its section by a plane perpendicular to the beam axis represents a rectangle in the angular coordinates $(\theta, \varphi)$, and the centre of it is determined by the position of the positron 3-momentum. The 
length of this rectangle along polar axis is equal to $2 \theta_{0}$, and along an azimutal one $-2 \Phi$. Their values are

$$
\theta_{0}=0.051 / 16, \quad \Phi=3 \pi / 32
$$

The cuts, for the cases when a photon and positron form or do not form cluster CALO2, are very simply formulated in the terms of the positron and photon variables, namely

$$
\left|\theta_{+}-\theta_{\gamma}\right|<\theta_{0}, \quad 1>\cos \varphi_{\gamma}>\cos \Phi, \quad\left(\gamma, e^{+}\right) \in C L
$$

and also

$$
\begin{gathered}
\left|\theta_{+}-\theta_{\gamma}\right|>\theta_{0}, \quad 1>\cos \varphi_{\gamma}>-1 ; \\
\left|\theta_{0}-\theta_{\gamma}\right|<\theta_{0}, \quad-1<\cos \varphi_{\gamma}<\cos \Phi, \quad\left(\gamma, e^{+}\right) \notin C L,
\end{gathered}
$$

where $\theta_{\gamma}=\vec{k}^{\perp} /(\varepsilon(1-x))$, and $\varphi_{\gamma}$ is the angle between two-dimensional vectors $\vec{k}^{\perp}$ and $\vec{q}_{2}^{\perp}$.

It is necessary to express the conditions (24) and (25) in terms of electron and positron variables $z, z_{1}$ and $\varphi$. Note in this case, that the law of the transverse momentum conservation leads to the following relations:

$$
\cos \varphi_{\gamma}=-\frac{\theta_{-} \cos \varphi+x \theta_{+}}{(1-x) \theta_{\gamma}}, \quad \theta_{\gamma}=\frac{1}{1-x} \sqrt{\theta_{-}^{2}+x^{2} \theta_{+}^{2}+2 x \theta_{+} \theta_{-} \cos \varphi} .
$$

Using these relations we take for the case when photon and positron form a cluster

$$
\begin{gathered}
A<\varphi<B, \quad A=\max \left[-1=\frac{(1-x)^{2}\left(\theta_{+}-\theta_{0}\right)^{2}-\left(\theta_{-}-x \theta_{+}\right)^{2}}{2 x \theta_{+} \theta_{-}}, \quad-1\right], \\
B=\min \left[-1+\frac{(1-x)^{2}\left(\theta_{+}+\theta_{0}\right)^{2}-\left(\theta_{-}-x \theta_{+}\right)^{2}}{2 x \theta_{+} \theta_{-}}, \cos (\Phi-\delta)\right], \sin \delta=\sqrt{\frac{x \theta_{+}}{\theta_{-}}} \sin \Phi .
\end{gathered}
$$

From Eq.(27) it follows that there are three different kinematic regions if photon and positron belong to the cluster CALO2. In the first region we have

$$
\begin{gathered}
-1+\frac{\left.(1-x)^{2}\left(\sqrt{z_{1}}-x \bar{\lambda}\right)^{2}-x^{2} \sqrt{z}-\sqrt{z_{1}}\right)^{2}}{2 x^{2} \sqrt{z z_{1}}}<\cos \varphi<-\cos (\Phi-\delta), \\
x^{2} z_{+}<z_{1}<x^{2} J_{+}^{2} .
\end{gathered}
$$

In the second one

$$
-1<\cos \varphi<-\cos (\Phi-\delta), \quad x^{2} z_{+}>z_{1}>x^{2} J_{-}^{2},
$$


and in the third region

$$
-1+\frac{\left.(1-x)^{2}\left(\sqrt{z_{1}}+x \bar{\lambda}\right)^{2}-x^{2} \sqrt{z}-\sqrt{z_{1}}\right)^{2}}{2 x^{2} \sqrt{z z_{1}}}>\cos \varphi>-1, \quad x^{2} z_{+}<z_{1}<x^{2} J_{+}^{2},
$$

where

$$
\begin{gathered}
J_{( \pm)}=\frac{1}{\beta}\left[\sqrt{z \beta-x^{2}(1-x)^{2} \bar{\lambda}^{2} \sin ^{2} \Phi} \pm(1-x) \bar{\lambda}\left(1-2 x \sin ^{2} \frac{\Phi}{2}\right)\right] \\
\beta=1-4 x(1-x) \sin ^{2} \frac{\Phi}{2}, \quad \bar{\lambda}=\frac{\theta_{0}}{\theta_{1}} .
\end{gathered}
$$

If the photon and positron do not form the cluster CALO2, then it is evident that

$$
-1<\cos \varphi<1, \quad z_{1}>x^{2} J_{+}^{2}, \quad z_{1}<x^{2} z_{-},
$$

and in the opposite case

$$
\begin{gathered}
-1+\frac{\left.(1-x)^{2}\left(\sqrt{z_{1}}-x \bar{\lambda}\right)^{2}-x^{2} \sqrt{z}-\sqrt{z_{1}}\right)^{2}}{2 x^{2} \sqrt{z z_{1}}}>\cos \varphi>-1, \quad-\cos (\Phi-\delta)<\cos \varphi<1, \\
x^{2} z_{+}<z_{1}<x^{2} J_{+}^{2} \\
-\cos (\Phi-\delta)<\cos \varphi<1, \quad x^{2} z_{+}>z_{1}>x^{2} J_{-}^{2} \\
-1+\frac{\left.(1-x)^{2}\left(\sqrt{z_{1}}+x \bar{\lambda}\right)^{2}-x^{2} \sqrt{z}-\sqrt{z_{1}}\right)^{2}}{2 x^{2} \sqrt{z z_{1}}}<\cos \varphi<1, \quad x^{2} z_{-}<z_{1}<x^{2} J_{-}^{2}
\end{gathered}
$$

Integration of Eq.(17) leads to the following result for the contribution to the part of the first order correction which depends on the shape and size of the cluster CALO2

$$
\begin{gathered}
\Sigma_{1 i}^{c l}=\frac{\alpha}{2 \pi} \int_{0}^{x_{c}} \frac{1+x^{2}}{1-x} d x \int \frac{d z}{z^{2}} \int d z_{1}\left[\left(\Psi_{1}+\widetilde{\Psi}_{1}\right) \Phi_{1}+\left(\Psi_{2}+\widetilde{\Psi}_{2}\right) \Phi_{2}+\right. \\
\left.+\left(\Psi_{3}+\widetilde{\Psi}_{3}\right) \Phi_{3}\right] \frac{2}{\pi}\left(\frac{1}{z_{1}-x z}+\frac{1}{z-z_{1}}\right) \\
\Sigma_{1 f}^{c l}=\frac{\alpha}{2 \pi} \int_{0}^{x_{c}} \frac{1+x^{2}}{1-x} d x\left\{\int \frac { d z } { z ^ { 2 } } \int d z _ { 1 } \left[\left(\Psi_{1}+\widetilde{\Psi}_{1}\right) F_{1}+\left(\Psi_{4}+\widetilde{\Psi}_{4}\right) F_{2}+\right.\right. \\
\left.+\left(\Psi_{3}+\widetilde{\Psi}_{3}\right) F_{3}\right] \frac{2}{\pi}\left(\frac{1}{z_{1}-x z}-\frac{1}{z_{1}-x^{2} z}\right)+ \\
+\int_{1}^{\rho_{3}^{2}} \frac{d z}{z^{2}}\left[\theta \left(\max \left(1, z_{2}^{(-)}-z\right)-\theta\left(z-\min \left(\left(\rho_{4}+\bar{\lambda}(1-x)\right)^{2}, \rho_{3}^{2}\right)\right] \widetilde{L}_{4}+\right.\right.
\end{gathered}
$$




$$
\begin{gathered}
+\int_{\tilde{a}^{2}}^{\tilde{b}_{0}^{2}} \frac{d z}{z^{2}}\left(\ln \left|\frac{x \rho_{2}^{2}-z}{\rho_{2}^{2}-z}\right|+l_{-}\right)+\int_{\max \left(1, z_{2}^{(-)}\right)}^{z_{4}^{(-)}} \frac{d z}{z^{2}} \ln \left|\frac{\left(x \rho_{4}^{2}-z\right)\left(J_{+}^{2}-z\right)}{\left(x J_{+}^{2}-z\right)\left(\rho_{4}^{2}-z\right)}\right|+ \\
\left.\int_{\rho_{2}^{2}}^{\min \left(\rho_{4}^{2}, z_{3}^{(-)}\right)} \frac{d z}{z^{2}} \ln \left|\frac{\left(x \rho_{3}^{2}-z\right)\left(J_{+}^{2}-z\right)}{\left(x J_{+}^{2}-z\right)\left(\rho_{3}^{2}-z\right)}\right|+\int_{a^{2}}^{\rho_{4}^{2}} \frac{d z}{z^{2}}\left(\ln \left|\frac{z-x}{1-z}\right|+l_{-}\right)\right\}
\end{gathered}
$$

where

$$
z_{i}^{( \pm)}=\left(\rho_{i} \pm(1-x) \bar{\lambda}\right)^{2}-4 x(1-x) \rho_{i}\left(\rho_{i} \pm \bar{\lambda}\right) \sin ^{2} \frac{\Phi}{2} .
$$

The $\Phi_{i}$ and $F_{i}$ functions, entering in Eqs.(35) and (36), are defined as follows

$$
\begin{gathered}
\Phi_{1}=\arctan Q_{i}^{(-)}-\arctan \eta, \quad \Phi_{2}=\arctan \eta^{-1}, \quad \Phi_{3}=\arctan \frac{1}{Q_{i}^{(+)}}, \\
F_{1}=\arctan \frac{1}{Q_{f}^{(-)}}, \quad F_{2}=\arctan \zeta, \quad F_{3}=\arctan \frac{1}{Q_{f}^{(+)}}, \quad \eta=R_{i} \cot \frac{\Phi-\delta}{2}, \\
\zeta=R_{f} \cot \frac{\Phi-\delta}{2}, \quad Q_{f}^{( \pm)}=\frac{R_{f}}{R_{i}} Q_{i}^{( \pm)}, \quad R_{i}=\frac{\left(\sqrt{z}-\sqrt{z_{1}}\right)^{2}}{z-z_{1}}, \quad R_{f}=\frac{\left(\sqrt{z_{1}}-x \sqrt{z}\right)^{2}}{z_{1}-x^{2} z}, \\
Q_{i}^{( \pm)}=R_{i} \sqrt{\frac{x^{2}\left(\sqrt{z}+\sqrt{z_{1}}\right)^{2}-(1-x)^{2}\left(\sqrt{z_{1}} \pm x \bar{\lambda}\right)^{2}}{(1-x)^{2}\left(\sqrt{z_{1}} \pm x \bar{\lambda}\right)^{2}-x^{2}\left(\sqrt{z}-\sqrt{z_{1}}\right)^{2}} .}
\end{gathered}
$$

The $\Psi_{i}$ and $\widetilde{\Psi}_{i}$ quantities are inserted in Eqs.(35) and (36) in order to indicate the integration limits over $z$ and $z_{1}$

$$
\begin{gathered}
\Psi_{1}=\left[\min \left(\rho_{4}^{2}, z_{3}^{(-)}\right), \rho_{2}^{2}\right]\left(x^{2} J_{+}^{2}, x^{2} z_{+}\right)+\left[b^{2}, z_{3}^{(-)}\right]\left(x^{2} \rho_{3}^{2}, x^{2} z_{+}\right) \\
\Psi_{2}=\left[\max \left(\rho_{2}^{2}, z_{1}^{(+)}\right), \rho_{2}^{2}\right]\left(x^{2} z_{+}, x^{2}\right)+\left[b^{2}, \max \left(\rho_{2}^{2}, z_{1}^{(+)}\right]\left(x^{2} z_{+}, x^{2} J_{-}^{2}\right)+\left[\rho_{4}^{2}, b^{2}\right]\left(x^{2} \rho_{3}^{2}, x^{2} J_{-}^{2}\right)\right. \\
\Psi_{3}=\left[a^{2}, \max \left(\rho_{2}^{2}, z_{1}^{(+)}\right)\right]\left(x^{2} J_{-}^{2}, x^{2}\right)+\left[\rho_{4}^{2}, a^{2}\right]\left(x^{2} J_{-}^{2}, x^{2} z_{-}\right) \\
\Psi_{4}=\left[\max \left(\rho_{2}^{2}, z_{1}^{(+)}\right), \rho_{2}^{2}\right]\left(x^{2} J_{+}^{2}, x^{2}\right)+\left[\min \left(\rho_{4}^{2}, z_{3}^{(-)}\right), \max \left(\rho_{2}^{2}, z_{1}^{(+)}\right]\left(x^{2} J_{+}^{2}, x^{2} J_{-}^{2}\right)+\right. \\
+\left[\rho_{4}^{2}, \min \left(\rho_{4}^{2}, z_{3}^{(-)}\right)\right]\left(x^{2} \rho_{3}^{2}, x^{2} J_{-}^{2}\right) \\
\widetilde{\Psi}_{1}=\left[\left(\rho_{2}^{2}-\bar{\lambda}(1-x)\right)^{2}, \max \left(1, z_{2}^{(-)}\right]\left(x^{2} J_{+}^{2}, x^{2} \rho_{2}^{2}\right)+\left[z_{4}^{(-)}, \tilde{a}_{0}\right]\left(x^{2} J_{+}^{2}, x^{2} z_{+}\right)+\right. \\
\left.\left[\left(\rho_{4}-\bar{\lambda}(1-x)\right)^{2}, z_{4}^{(-)}\right)\right]\left(x^{2} \rho_{4}^{2}, x^{2} z_{+}\right) \\
\left.\widetilde{\Psi}_{2}=\left[z_{2}^{(+)}, \tilde{a}_{0}^{2}\right]\left(x^{2} z_{+}, x^{2} \rho_{2}^{2}\right)+\left[\left(\rho_{4}-\bar{\lambda}(1-x)\right)^{2}, z_{2}^{(+)}\right)\right]\left(x^{2} z_{+}, x^{2} J_{-}^{2}\right)+ \\
+\left[\min \left(\rho_{3}^{2}, z_{4}^{(+)}\right),\left(\rho_{4}-\bar{\lambda}(1-x)\right)^{2}\right]\left(x^{2} \rho_{4}^{2}, x^{2} J_{+}^{2}\right) \\
\widetilde{\Psi}_{3}=\left[\left(\rho_{2}^{2}+\bar{\lambda}(1-x)\right)^{2}, z_{2}^{(+)}\right]\left(x^{2} J_{-}^{2}, x^{2} \rho_{2}^{2}\right)+\left[\tilde{b}_{0},\left(\rho_{2}^{2}+\bar{\lambda}(1-x)\right)^{2}\right]\left(x^{2} J_{+}^{2}, x^{2} z_{+}\right)
\end{gathered}
$$




$$
\begin{gathered}
\widetilde{\Psi}_{4}=\left[z_{2}^{(+)}, \max \left(1, z_{2}^{(-)}\right)\right]\left(x^{2} J_{+}^{2}, x^{2} \rho_{2}^{2}\right)+\left[z_{4}^{(-)}, z_{2}^{(+)}\right]\left(x^{2} J_{+}^{2}, x^{2} J_{-}^{2}\right)+ \\
+\left[\min \left(\rho_{3}^{2}, z_{4}^{(+)}\right), z_{4}^{(-)}\right]\left(x^{2} \rho_{4}^{2}, x^{2} J_{-}^{2}\right) .
\end{gathered}
$$

It is necessary to replace $\lambda$ by $\bar{\lambda}$ in the used here quantities $a, b, a_{0}, b_{0}$. As before, in the case of symmetrical wide detectors, in Eqs.(35) and (36) one must replace $\rho_{4}$ by $\rho_{3}$ and $\rho_{2}$ by 1 and simultaneously put to zero those terms where the upper integration limit over $z$ becomes less than bottom one.

\section{Leading second- and third-order corrections}

As we have seen in the previous Sections, the large logarithm $L$ enters only in the universal part of the radiative correction. Such situation takes place also for leading contributions in the higher orders of the perturbation theory. Therefore, the analytical formulae for high-order leading radiative corrections are universal. They are applicable for both CALO1 and CALO2 event selections.

In order to obtain the leading contributions in the second and third orders we use the method based on the electron structure functions in the singlet and nonsinglet channels [16] and effective coupling constant [7]

$$
D\left(x, \alpha_{e f f}\right)=D^{N S}\left(x, \alpha_{e f f}\right)+D^{S}\left(x, \alpha_{e f f}\right),
$$

where the effective coupling constant is defined as the integral of the running electromagnetic constant in one-loop approximation

$$
\frac{\alpha_{e f f}}{2 \pi}=\int_{0}^{L} \frac{\alpha d t}{2 \pi\left(1-\frac{\alpha t}{3 \pi}\right)}=\frac{3}{2} \ln \left(1-\frac{\alpha L}{3 \pi}\right)^{-1} .
$$

The iterative form of the nonsinglet component of the structure function can be presented as

$$
\begin{gathered}
D^{N S}\left(x, \alpha_{e f f}\right)=\delta(1-x)+\sum_{k=1}^{\infty} \frac{1}{k !}\left(\frac{\alpha_{\text {eff }}}{2 \pi}\right)^{k} P_{1}(x)^{\otimes k}, \\
\underbrace{P_{1}(x) \otimes \cdots \otimes P_{1}(x)}_{k}=P_{1}(x)^{\otimes k}, \quad P_{1}(x) \otimes P_{1}(x)=\int_{x}^{1} P_{1}(t) P_{1}\left(\frac{x}{t}\right) \frac{d t}{t} .
\end{gathered}
$$

The singlet component of the electron structure function including third-order contribution can be written as follows

$$
D^{S}\left(x, \alpha_{e f f}\right)=\frac{1}{2 !}\left(\frac{\alpha_{e f f}}{2 \pi}\right)^{2} R(x)+\frac{1}{3 !}\left(\frac{\alpha_{e f f}}{2 \pi}\right)^{3}\left[2 P_{1} \otimes R(x)-\frac{2}{3} R(x)\right],
$$




$$
R(x)=\frac{1-x}{3 x}\left(4+7 x+4 x^{2}\right)+2(1+x) \ln x .
$$

The nonsinglet component of the electron structure function describes the photon emission and electron-positron pair production neglecting the final-electron identity, whereas the singlet one just responds for the identity effect.

With the required accuracy the electron structure function reads

$$
\begin{gathered}
D(x, L)=\delta(1-x)+\frac{\alpha L}{2 \pi} P_{1}(x)+\frac{1}{2}\left(\frac{\alpha L}{2 \pi}\right)^{2} G(x)+\frac{1}{3}\left(\frac{\alpha L}{2 \pi}\right)^{3} F(x), \\
G(x)=P_{2}(x)+\frac{2}{3} P_{1}(x)+R(x), \quad F(x)=\frac{1}{2} P_{3}(x)+P_{2}(x)+\frac{4}{9} P_{1}(x)+\frac{2}{3} R(x)+R^{x}(x), \\
P_{i}(x)=P_{1}^{\otimes_{i}}, \quad R^{p}(x)=P_{1} \otimes R(x) .
\end{gathered}
$$

The functions $P_{2}(x), P_{3}(x)$ and $R^{p}(x)$, entering in the right side of Eq.(42,) can be written as follows [7]

$$
\begin{gathered}
R^{p}(x)=\left[\frac{3}{2}+2 \ln (1-x)\right] R(x)+(1+x)\left[4 L_{i 2}(1-x)-\ln ^{2} x\right]+\frac{1}{3}(-9-3 x+ \\
\left.+8 x^{2}\right) \ln x+\frac{2}{3}\left(-\frac{3}{x}-8+8 x+3 x^{2}\right) \\
P_{i}(x)=\Delta_{i} \delta(1-x)+\Theta_{i}(x) \theta(1-x-\Delta), \quad i=2, \quad 3 \\
\Delta_{2}=\left(2 \ln \Delta+\frac{3}{2}\right)^{2}-4 \zeta_{2}, \Delta_{3}=\left(2 \ln \Delta+\frac{3}{2}\right)^{3}-12 \zeta_{2}\left(2 \ln \Delta+\frac{3}{2}\right)+16 \zeta_{3}, \\
\Theta_{2}(x)=2\left[\frac{1+x^{2}}{1-x}\left(\ln \frac{(1-x)^{2}}{x}+\frac{3}{2}\right)+\frac{1}{2}(1+x) \ln x-1+x\right] \\
\Theta_{3}(x)=12 \frac{1+x^{2}}{1-x}\left[\ln \frac{1-x}{x} \ln (1-x)+\frac{1}{6} \ln 2 x+\frac{3}{4} \ln \frac{(1-x)^{2}}{x}+\frac{9}{16}-\zeta_{2}\right]-3(1-x)[1+ \\
+4 \ln (1-x)]+\frac{3}{2}(5-3 x) \ln x+6(1+x)\left[\frac{1}{4} \ln x \ln \frac{(1-x)^{4}}{x}+L_{i 2}(1-x)\right] .
\end{gathered}
$$

The functions $P_{i}(x)$ satisfy the condition

$$
\int_{0}^{1} P_{i}(x) d x=0,
$$

which is nothing but the statement of the Lee-Nauenberg theorem [21] for the leading radiative corrections in the singlet channel in terms of the electron structure functions. 
The factorization form of the differential cross-section in the frame work of the impact representation [23] determines the leading contribution to the radiative correction in the case of calorimeter event selection in the following form

$$
\Sigma^{L}=\int_{0}^{\infty} \frac{d z}{z^{2}} \int_{x_{c}}^{1} d x_{1} \int_{x_{c} / x_{1}}^{1} d x_{2} D\left(x_{1}, L\right) D\left(x_{2}, L\right) \Delta_{31}^{\left(x_{1}\right)} \Delta_{42}^{\left(x_{2}\right)} .
$$

The integrand in Eq.(43) includes only those $\theta$-functions which depend on $x_{1}$ and $x_{2}$. It corresponds to account for the initial-state emission. The final-state emission does not produce the leading contribution according to the mentioned above Lee-Nauenberg theorem.

Combining Eqs.(42) and (43) we obtain the leading contribution in the second and third orders

$$
\begin{gathered}
\Sigma_{2}^{L}=\frac{\alpha^{2}}{8 \pi^{2}} \int_{0}^{\infty} \frac{d z}{z^{2}} L^{2}\left\{\int_{x_{c}}^{1}\left[\left(\Delta_{42}^{(x)} \Delta_{31}+\Delta_{31}^{(x)} \Delta_{42}\right) G(x)+2 \int_{x_{c} / x}^{1} d x_{1} P_{1}(x) P_{1}\left(x_{1}\right) \Delta_{31}^{(x)} \Delta_{42}^{\left(x_{1}\right)}\right] d x\right\} \\
\Sigma_{3}^{L}=\frac{\alpha^{3}}{8 \pi^{3}} \int_{0}^{\infty} \frac{d z}{z^{2}} L^{3}\left\{\int _ { x _ { c } } ^ { 1 } \left[\frac{1}{3}\left(\Delta_{42}^{(x)} \Delta_{31}+\Delta_{31}^{(x)} \Delta_{42}\right) F(x)+\right.\right. \\
\left.+\frac{1}{2} \int_{x_{c} / x}^{1} d x_{1} P_{1}(x) G\left(x_{1}\right)\left(\Delta_{31}^{(x)} \Delta_{42}^{\left(x_{1}\right)}+\Delta_{31}^{\left(x_{1}\right)} \Delta_{42}^{(x)}\right] d x\right\}
\end{gathered}
$$

In order to eliminate the $\theta$ - functions, entering in (44) and (45), and place specific limits, the additional work is needed. Omitting all intermediate calculations let us write the second-order leading correction as follows

$$
\Sigma_{2}^{L}=\Sigma_{2(-)}^{L}+\Sigma_{2(+)}^{L}+\Sigma_{\gamma}^{\gamma}
$$

where the first term on the rihgt side of Eq.(46) is caused due to the emission of the real and virtual photons as well as electron-positron pair production by an electron, the second term - by the positron, and the third one - due to the emission of one photon by an electron and one photon by a positron:

$$
\begin{gathered}
\Sigma_{2(-)}^{L}=\frac{\alpha^{2}}{8 \pi^{2}}\left[\int_{\rho_{2}^{2}}^{\rho_{4}^{2}} \frac{d z}{z^{2}} L^{2} A-\int_{m_{23}}^{\rho_{4}^{2}} \frac{d z}{z^{2}} L^{2} B\left(\frac{\sqrt{z}}{\rho_{3}}\right)\right], \\
\left.\Sigma_{2(+)}^{L}=\frac{\alpha^{2}}{8 \pi^{2}}\left[\int_{\rho_{2}^{2}}^{\rho_{4}^{2}} \frac{d z}{z^{2}} L^{2} A-\int_{m_{14}}^{\rho_{4}^{2}} \frac{d z}{z^{2}} L^{2} B\left(\frac{\sqrt{z}}{\rho_{4}}\right)\right]+\int_{m_{12}}^{\rho_{2}^{2}} \frac{d z}{z^{2}} L^{2} B\left(\frac{\sqrt{z}}{\rho_{2}}\right)\right],
\end{gathered}
$$




$$
\begin{aligned}
\Sigma_{\gamma}^{\gamma}= & \frac{\alpha^{2}}{4 \pi^{2}}\left\{-\int_{\rho_{2}^{2}}^{\rho_{4}^{2}} \frac{d z}{z^{2}} L^{2} F_{2}\left(x_{c}\right)-\int_{m_{23}}^{\rho_{4}^{2}} \frac{d z}{z^{2}} L^{2} F_{g}\left(\frac{\sqrt{z}}{\rho_{3}}, x_{c}\right)\right]-\int_{m_{14}}^{\rho_{4}^{2}} \frac{d z}{z^{2}} L^{2} F_{g}\left(\frac{\sqrt{z}}{\rho_{4}}, x_{c}\right)+ \\
& +\int_{m_{12}}^{\rho_{2}^{2}} \frac{d z}{z^{2}} L^{2} F_{g}\left(\frac{\sqrt{z}}{\rho_{2}}, x_{c}\right)+\int_{x_{c} \rho_{3} \rho_{4}}^{\rho_{4}^{2}} \frac{d z}{z^{2}} L^{2}\left[F_{g}\left(\frac{\sqrt{z}}{\rho_{4}}, \frac{x_{c} \rho_{3}}{\sqrt{z}}\right)+C\left(\frac{\sqrt{z}}{\rho_{3}}, \frac{\sqrt{z}}{\rho_{4}}\right)\right]+ \\
& \int_{x_{c} \rho_{2}}^{1} \frac{d z}{z^{2}} L^{2}\left[F_{g}\left(\sqrt{z}, \frac{x_{c} \rho_{2}}{\sqrt{z}}\right)+C\left(\frac{\sqrt{z}}{\rho_{2}}, \sqrt{z}\right)\right]-\int_{x_{c} \rho_{4}}^{\frac{1}{z^{2}}} L^{2}\left[F_{g}\left(\frac{\sqrt{z}}{\rho_{4}}, \frac{x_{c}}{\sqrt{z}}\right)+\right. \\
& \left.\left.+C\left(\sqrt{z}, \frac{\sqrt{z}}{\rho_{4}}\right)\right]-\int_{x_{c} \rho_{3} \rho_{2}}^{\rho_{2}^{2}} \frac{d z}{z^{2}} L^{2}\left[F_{g}\left(\frac{\sqrt{z}}{\rho_{3}}, \frac{x_{c} \rho_{2}}{\sqrt{z}}\right)+C\left(\frac{\sqrt{z}}{\rho_{2}}, \frac{\sqrt{z}}{\rho_{3}}\right)\right]\right\},
\end{aligned}
$$

where

$$
\begin{gathered}
m_{i j}=\max \left(x_{c}^{2} \rho_{j}^{2}, \rho_{i}^{2}\right), A=-\int_{0}^{x_{c}}\left(P_{2}(x)+\frac{2}{3} P_{1}(x)\right) d x+\int_{x_{c}}^{1} R(x) d x, \quad B(y)=\int_{x_{c}}^{y} G(x) d \\
F_{2}(x)=\int_{0}^{x} P_{2}(y) d y=-2 x-\frac{x^{2}}{4}+\left(x+\frac{x^{2}}{2}\right) \ln \frac{x^{3}}{(1-x)^{4}}+4 \ln (1-x) \ln \frac{x}{1-x}+4 L_{i 2}(x) \\
F_{g}(x, y)=F_{g}(x)-F_{g}(y), \quad x_{g}^{2} \\
F_{g}(x)=\int P_{1}(x) g\left(\frac{x_{c}}{x}\right) d x=-\frac{x_{c}^{2}}{x}+\left(2 x+x^{2}\right) \ln x+\left(x_{c}+\frac{x_{c}^{2}}{2}\right) \ln \frac{x}{(1-x)^{2}}+\left(2 x_{c}+\frac{x_{c}}{2}\right. \\
\left.-2 x-\frac{x^{2}}{2}\right) \ln \left(1-x_{c}\right)+4 L_{i 2}(x)+4 L_{i 2}\left(\frac{1-x}{1-x_{c}}\right), \quad x_{c}<x<1 \\
C(x, y)=g(x)\left[g(y)-g\left(\frac{x_{c}}{x}\right)\right], \\
g(x)=-\int P_{1}(x) d x=x+\frac{x^{2}}{2}+2 \ln (1-x), \quad x<1 .
\end{gathered}
$$

By analogy with Eq.(46) the third-order leading correction can be written as follows

$$
\Sigma_{3}^{L}=\Sigma_{0}^{3}+\Sigma_{3}^{0}+\Sigma_{1}^{2}+\Sigma_{2}^{1}
$$

where the upper index shows the number of real and virtual particles emitted by an electron and the bottom one - by a positron. For the case when three additional particles are emitted by one of the fermions, the corresponding contribution to $\Sigma_{3}^{L}$ reads

$$
\Sigma_{0}^{3}+\Sigma_{3}^{0}=\left(\frac{\alpha}{2 \pi}\right)^{3}\left\{\int_{\rho_{2}^{2}}^{\rho_{4}^{2}} \frac{d z}{z^{2}} L^{3}\left[-2 \int_{0}^{x_{c}} F_{p}(x) d x+2 \int_{x_{c}}^{1} F_{r}(x) d x\right]-\right.
$$


$\left.-\int_{m_{23}}^{\rho_{4}^{2}} \frac{d z}{z^{2}} L^{3} \int_{x_{c}}^{\sqrt{z} / \rho_{3}} F_{c}(x) d x-\int_{m_{14}}^{\rho_{4}^{2}} \frac{d z}{z^{2}} L^{3} \int_{x_{c}}^{\sqrt{z} / \rho_{4}} F_{c}(x) d x+\int_{m_{12}}^{\rho_{2}^{2}} \frac{d z}{z^{2}} L^{3} \int_{x_{c}}^{\sqrt{z} / \rho_{2}} F_{c}(x) d x\right\}$

$$
\begin{gathered}
F_{p}(x)=\frac{1}{6} P_{3}(x)+\frac{1}{3} P_{2}(x)+\frac{4}{27} P_{1}(x), \\
F_{r}(x)=\frac{2}{9} R(x)+\frac{1}{3} R^{p}(x), \quad F_{c}(x)=\frac{1}{3} F(x) .
\end{gathered}
$$

In the case when both fermions radiate simultaneously, we have

$$
\begin{aligned}
\Sigma_{1}^{2}+\Sigma_{2}^{1} & =\left(\frac{\alpha}{2 \pi}\right)^{3}\left\{\int_{\rho_{2}^{2}}^{\rho_{4}^{2}} \frac{d z}{z^{2}} L^{3}\left[-\int_{0}^{x_{c}}\left(P_{3}(x)+\frac{2}{3} P_{2}(x)\right) d x+\int_{x_{c}}^{1} R^{p}(x) d x\right]-\right. \\
-\int_{m_{23}}^{\rho_{4}^{2}} \frac{d z}{z^{2}} L^{3} & \int_{x_{c}}^{\sqrt{z} / \rho_{3}} H\left(x, x_{c}\right) d x-\int_{m_{14}}^{\rho_{4}^{2}} \frac{d z}{z^{2}} L^{3} \int_{x_{c}}^{\sqrt{z} / \rho_{4}} H\left(x, x_{c}\right) d x+\int_{m_{12}}^{\rho_{2}^{2}} \frac{d z}{z^{2}} L^{3} \int_{x_{c}}^{\sqrt{z} / \rho_{2}} H\left(x, x_{c}\right), \\
& +\int_{x_{c} \rho_{3} \rho_{4}}^{\rho_{4}^{2}} \frac{d z}{z^{2}} L^{3}\left[\int_{x_{c} \rho_{4} / \sqrt{z}}^{\sqrt{z} / \rho_{3}} d x P_{1}(x) N\left(\frac{x_{c}}{x} ; \frac{\sqrt{z}}{\rho_{4}}\right) d x+\left(\rho_{3} \leftrightarrow \rho_{4}\right)\right]+ \\
& +\int_{x_{c} \rho_{2}}^{1} \frac{d z}{z^{2}} L^{3}\left[\int_{x_{c} \rho_{2} / \sqrt{z}}^{\sqrt{z} / 1} d x P_{1}(x) N\left(\frac{x_{c}}{x} ; \frac{\sqrt{z}}{\rho_{2}}\right) d x+\left(\rho_{2} \leftrightarrow 1\right)\right]- \\
& -\int_{x_{c} \rho_{2} \rho_{3}}^{\rho_{2}^{2}} L^{3}\left[\int_{x_{c} \rho_{2} / \sqrt{z}}^{\sqrt{z} / \rho_{3}} d x P_{1}(x) N\left(\frac{x_{c}}{x} ; \frac{\sqrt{z}}{\rho_{2}}\right) d x+\left(\rho_{3} \leftrightarrow \rho_{2}\right)\right]- \\
& \left.-\int_{x_{c} \rho_{4}}^{1} \frac{d z}{z^{2}} L^{3}\left[\int_{x_{c} \rho_{4} / \sqrt{z}}^{\sqrt{z} / 1} d x P_{1}(x) N\left(\frac{x_{c}}{x} ; \frac{\sqrt{z}}{\rho_{4}}\right) d x+\left(\rho_{4} \leftrightarrow 1\right)\right]\right\}
\end{aligned}
$$

where

$$
\begin{gathered}
H\left(x, x_{c}\right)=P_{1}(x)\left[\frac{1}{2} f\left(\frac{x_{c}}{x}\right)+\frac{2}{3} g\left(\frac{x_{c}}{x}\right)+\frac{1}{2} r\left(\frac{x_{c}}{x}\right)\right]+\frac{1}{2} g\left(\frac{x_{c}}{x}\right)\left(P_{2}(x)+R(x)\right), \\
N(x ; y)=N(x)-N(y), \quad N(y)=\frac{1}{2} f(y)+\frac{1}{3} g(y)+\frac{1}{2} r(y), \\
f(y)=-F_{2}(y), \quad r(y)=\int_{y}^{1} R(x) d x=-\frac{22}{9}+y+y^{2}+\frac{4}{9} y^{3}-\left(\frac{4}{3}+2 y+y^{2}\right) \ln y .
\end{gathered}
$$

When writing the formulae of this Section, we represent the restrictions on the angles and energies of the detected particles with the help of definite integrals using such relations as, for example,

$$
\int \theta_{4} \bar{\theta}_{4}^{(x)} \bar{\theta}_{3}^{\left(x_{1}\right)} d z d x d x_{1}=\int_{x_{c} \rho_{3}}^{\rho_{4}^{2}} d z \int_{x_{c} \rho_{3} / \sqrt{z}}^{\sqrt{z} / \rho_{4}} d x \int_{x_{c} / x}^{\sqrt{z} / \rho_{3}} d x_{1} .
$$


It is necessary to bear in mind that the upper limit of the integration over the variable $z$ always must be greater than bottom one. In opposite case the integral must be putted to zero.

\section{$6 \quad$ Numerical results}

At LEP1 conditions the limiting angles of the ring-shaped detectors have different values for various versions of event selection. In the cases of BARE1 and CALO1 the wide detector has

$$
\theta_{1}=0.024, \quad \theta_{3}=0.058,
$$

and narrow one -

$$
\Theta_{2}=\theta_{1}+h, \quad \theta_{4}=\theta_{3}-h, \quad h=\frac{0.017}{8} .
$$

The wide detector $(\mathbf{w w})$ for the event selection CALO2 coinsides with the narrow one (nn) for the case BARE1, whereas the limiting angles of the narrow detector are determined as follows

$$
\theta_{2}=\theta_{1}+2 h, \quad \theta_{4}=\theta_{3}-4 h .
$$

The Born cross-section, determined by the formula (4), is equal to

$\sigma_{B}=175.922 \mathbf{n b}$ for $\mathbf{w} \mathbf{w}$ BARE1 CALO1,

$\sigma_{B}=139.971 \mathbf{n b}$ for $\mathbf{w} \mathbf{w}$ CALO2, $\mathbf{n n}$ BARE1 and CALO2,

$\sigma_{B}=103.299 \mathbf{n b} \mathbf{n n}$ CALO2.

The results of our calculations of the radiative QED corrections are presented in the Tables I-III, where the vacuum polarization is switched off. For the comparison we also present numbers in the Tables I and III, that are obtained with the help of the MC-generator BHLUMI using the exponentiated Yennie-FrautchiSuura factor for the higher-order corrections. 


\begin{tabular}{|ccccc|}
\hline$x_{c}$ & bhlumi ww & ww & nn & wn \\
\hline \multicolumn{5}{c|}{ calo1 } \\
\hline 0.1 & 166.329 & 166.285 & 131.032 & 134.270 \\
0.3 & 166.049 & 166.006 & 130.833 & 134.036 \\
0.5 & 165.287 & 165.244 & 130.416 & 133.466 \\
0.7 & 161.794 & 161.749 & 128.044 & 130.542 \\
0.9 & 149.925 & 149.866 & 118.822 & 120.038 \\
\hline \multicolumn{5}{c}{ calo2 } \\
\hline 0.1 & 131.032 & 130.997 & 94.666 & 98.354 \\
0.3 & 130.739 & 130.705 & 94.491 & 98.127 \\
0.5 & 130.176 & 130.141 & 94.177 & 97.720 \\
0.7 & 127.528 & 127.491 & 92.981 & 95.874 \\
0.9 & 117.541 & 117.491 & 86.303 & 87.696 \\
\hline
\end{tabular}

Table I.SABH cross-section at LEP1 conditions with first- order correction. Vacuum polarization is switched off

From the Table I one can see, that at LEP1 conditions, the SABH cross-section including the first-order correction, obtained by the means of BHLUMI generator systematically exceeds our results in all interval of values for parameter $x_{c}$. The relative difference amounts to about $0.03 \%$. The possible reason of this defference, as it was noted in Section 2, is that we neglect the contributions proportional to $\theta_{1}^{2}$ when calculating the radiative corrections. Within the accuracy of $0.1 \%$ the contribution of the omitted by us terms is insignificant. But it requires the additional systematic investigations if the accuracy will be $0.05 \%$ (and it had actually been achieved in the laboratory L3 [24]).

The absolute values of the second- and third-order leading corrections (in $\mathbf{n b}$ are presented in the Table II. The second-order correction is divided into the contributions related to the electron-positron pair production and double-photon emission. One can see that photonic corrections dominate in the second order of the perturbation theory. The third-order correction includes the contributions due to three-photon emission and pair production accompanied by single-photon emission. 


\begin{tabular}{|ccccccc|}
\hline \multicolumn{4}{|c|}{ calo1 } & \multicolumn{4}{c|}{ calo2 } \\
\hline$x_{c}$ & ww & nn & wn & ww & nn & wn \\
\hline \multicolumn{4}{|c|}{ correction due to pair productiion } \\
\hline 0.1 & -0.046 & -0.045 & -0.024 & -0.045 & -0.047 & -0.024 \\
0.3 & -0.046 & -0.045 & -0.024 & -0.045 & -0.047 & -0.024 \\
0.5 & -0.048 & -0.046 & -0.025 & -0.046 & -0.047 & -0.024 \\
0.7 & -0.069 & -0.059 & -0.042 & -0.059 & -0.051 & -0.036 \\
0.9 & -0.137 & -0.111 & -0.102 & -0.111 & -0.085 & -0.075 \\
\hline \multicolumn{6}{|c|}{ second order photonic correction } \\
\hline 0.1 & 0.788 & 0.708 & 0.302 & 0.708 & 0.668 & 0.255 \\
0.3 & 0.680 & 0.634 & 0.195 & 0.634 & 0.627 & 0.187 \\
0.5 & 0.474 & 0.487 & 0.005 & 0.487 & 0.554 & 0.073 \\
0.7 & 0.293 & 0.317 & -0.164 & 0.317 & 0.396 & -0.092 \\
0.9 & 0.866 & 0.738 & 0.373 & 0.738 & 0.628 & 0.271 \\
\hline \multicolumn{6}{|c|}{ third order correction } \\
\hline 0.1 & -0.041 & -0.036 & -0.002 & -0.036 & -0.034 & -0.001 \\
0.3 & -0.046 & -0.040 & -0.007 & -0.040 & -0.037 & -0.003 \\
0.5 & -0.044 & -0.039 & -0.006 & -0.039 & -0.037 & -0.005 \\
0.7 & -0.023 & -0.022 & 0.012 & -0.022 & -0.027 & 0.008 \\
0.9 & 0.021 & 0.013 & 0.049 & 0.013 & 0.002 & 0.038 \\
\hline
\end{tabular}

Table II Absolute values of second- and third-order corrections to SABH cross-section at LEP1 conditions (in $\mathbf{n b )}$

In the Table III we present the total SABH cross-section at LEP1 with account for all corrections calculated in this paper and also the results of the corresponding calculations made with the help of the MC-generator BHLUMI.

As to comparison of our calculations and MC-generator BHLUMI ones for the radiative corrections in the second and third orders, it is necessary to note that BHLUMI results are based on the exponentiated form of electron structure function whereas our ones - on the iterative form. The corresponding effect due to these different forms increases at large values of parameter $x_{c}$ as one can see from the Table III.

For more efficient comparison, it is necessary to have either the analytical calculations with the exponential form of electron structure functions or $\mathrm{MC}-$ calculations that do not use the exponentiation. For MC generator BHLUMI such calculations exist in the case of BARE1 event selection [3]. The corresponding 
comparison have been recently done and the agreement has been obtained at the very high level [25].

\begin{tabular}{|lclllll|}
\hline \multicolumn{4}{|c|}{ calo1 } & \multicolumn{4}{c|}{ calo2 } \\
\hline$x_{c}$ & ww & nn & wn & ww & nn & wn \\
\hline \multicolumn{4}{|c|}{ total cross-section (analytical calculation) } \\
\hline 0.1 & 166.968 & 131.659 & 134.546 & 131.624 & 95.253 & 98.584 \\
0.3 & 166.594 & 131.382 & 134.200 & 131.254 & 95.032 & 98.285 \\
0.5 & 165.626 & 130.818 & 133.440 & 130.543 & 94.647 & 97.764 \\
0.7 & 161.950 & 128.280 & 130.348 & 127.727 & 93.299 & 95.758 \\
0.9 & 150.616 & 119.462 & 120.358 & 118.131 & 86.848 & 87.930 \\
\hline \multicolumn{6}{|c|}{ total cross-section (bhlumi-generator) } \\
\hline 0.1 & 167.203 & 131.835 & 95.458 & 98.834 \\
0.3 & 166.795 & 131.450 & 95.233 & 98.539 \\
0.5 & 165.830 & 130.727 & 94.841 & 98.020 \\
0.7 & 162.237 & 127.969 & 93.520 & 96.054 \\
0.9 & 151.270 & 118.792 & 87.359 & 88.554 \\
\hline
\end{tabular}

Table III Total SABH cross-section at LEP1 conditions

The authors are grateful to A.Arbuzov, E.Kuraev and L.Trentadue for fruitful discussions and critical remarks. One of us (N.P.M.)would like to thank S.Jadach, B.Ward, G.Montagna and B.Pietrzyk for discussion of the results of this paper. This work was supported in part by INTAS Grant 93-1867 ext.

\section{References}

1. G. Barbiellini et al. L. Trentadue (conv.), Neutrino Counting in Z Physics at LEP; G. Altarelli, R. Kleiss, G. Verzegnassi, CERN Yellow Report 89-08 ; CERN Yellow Report 95-03 , Part I : "Electroweak Physics".

2. B. Pietrzyk, Preprint LAPP-EXP-94.18. Invited Talk on International Symposium on Radiative Corrections: Status and Outlook, Galtinburg, Tennessee, USA, June 1994, edited by Ward B.W.L. (World Scientific, Singapore, 1995) ;

LEP Electroweak Working Group. CERN Report LEPEWWG/95-02 ; LEP Collaborations, 1995, Collaboration notes: ALEPH 95-093 PHYSICS 95-086 
; DELPHI 95-137 PHYS 562 ; L3 Note 1814 ; OPAL Technical Note TN 312,1 August 1995.

3. H. Anlauf et al., Events Generator for Bhabha Scattering. In : Conveners: S. Jadach, O. Nicrosini. CERN Yellow Report 96-01, V.2. P.229. ; CERN Yellow Report 95-03 , Part III: "Small Angle Bhabha Scattering".

4. S. Jadach, E. Richter-Was E., B.F.L. Ward, Z. Was, Comput. Phys. Commun. 70 (1992) 305.

5. G. Montagna et al., Comput. Phys. Commun. 76 (1993) 328 .

M. Cassiori, G. Montagna, F. Piccinini, Comput. Phys. Commun. 90 (1995) 301.

G. Montagna et al., Nucl. Phys. bf B 401 (1993) 3.

6. S. Jadach, E. Richter-Was, B.F.L. Ward, Z. Was, Phys. Lett. B 353 (1995); 349,362 .

S. Jadach, M. Melles, B.F.L. Ward, S.A. Yost, Phys. Lett. B 377 (1996) 168.

7. S. Jadach M. Skrzypek, B.F.L. Ward, Phys. Rev. D 47 (1993) 3733 .

S. Jadach, E. Richter-Was, B.F.L. Ward, Z. Was, Phys. Lett. B 260 (1991) 438.

8. W. Beenakker, F.A. Berends, S.C. van der Marck, Nucl. Phys. B 355 (1991) 281.

W. Beenakker, B. Pietrzyk, Phys. Lett. B 304 (1993) 366.

9. M. Gaffo, H. Czyz, E. Remiddi, Nuovo Cim. A 105 (1992) 271 ; Int. J. Mod. Phys. 4 (1993) 591; Phys. Lett. B 327 (1994) 369.

10. G. Montagna, O. Nicrosini, F. Piccinini, Preprint FNT/T-96/8 .

11. A.B. Arbuzov et al., CERN Yellow Report 95-03 369.

12. A.B. Arbuzov et al., Nucl. Phys. B 485 (1997) 457.

13. A.B. Arbuzov, E.A. Kuraev, N.P. Merenkov, L. Trentadue, Zh.Eksp.Teor.Fiz. 108 (1995) 1164; Preprint CERN-TH/95-241, JINR-E2-95-110. 
14. N.P. Merenkov et al., Acta Phys. Pol. B 28 (1997) 491.

N.P. Merenkov, JETP 85 (1997) 217;

N.P. Merenkov, JETP Lett. 65 (1997) 235.

15. D.R. Yennie, S.C. Frautchi, H. Suura, Ann. Phys. 13 (1961) 379.

16. L.N. Lipatov, Sov. Journ. Nucl. Phys. 20 (1974) 121.

G. Altarelli, G. Parisi, Nucl. Phys. B 126 (1977) 298.

M. Skrzypek, Acta Phys. Pol. B 23 (1992) 135.

17. M. Gell-Mann, F. Low, Phys. Rev. 95 (1954) 1300.

18. V.G. Gorshkov, Usp. Fiz. Nauk 110 (1973) 45 .

V.N. Baier, S.A. Kheifez, Zh. Eksp. Teor. Fiz 40 (1961) 613 .

V.G. Gorshkov, V.N. Gribov, G.V. Frolov, Zh. Eksp. Teor. Fiz. 51 (1966) 1093 .

V.G. Gorshkov, V.N. Gribov, L.N. Lipatov, V.G. Frolov, Yad. Fiz. 6 (1967) 129, 361.

19. F.A. Berends et al., Nucl. Phys. B 57 (1973) 371.

E.A. Kuraev, G.V. Meledin, Nucl. Phys. B 122 (1977) 485.

20. S. Jadach, B.F.L. Ward, Phys. Rev. D 40 (1989) 3582.

21. T.D. Lee, M. Nauenberg, Phys. Rev. B 133 (1964) 1549.

22. N.P. Merenkov, Yad. Fiz. 48 (1988) 1782: 50 (1989) 750.

23. H. Cheng, T.T. Wu, Phys. Rev. Lett. 23 (1969) 670 .

V.G. Zima, N.P. Merenkov, Yad. Fiz. 25 (1976) 998.

V.N. Baier, V.S. Fadin, V. Khoze, E.A. Kuraev, Phys. Rep. 78 (1981) 294.

24. I.C. Brock et al., Preprint CERN-PPE/96-89' CMU-HEP/96-04.

25. A.B. Arbuzov et al., Phys. Lett B 399 (1997) 324. 